\title{
How Individuals Influence Supply Chain Management Performance?
}

\author{
Chen-Huei Chou \\ College of Charleston \\ 66 George Street \\ Charleston, SC, USA
}

\begin{abstract}
Supply chain management has been an important field in business operations. Due to the popularity of electronic commerce and mobile commerce, the supply chain field has been evolved to another level. Higher level of automation and use of computerized software have been deployed. Human intervention still cannot be avoided. Rather, human interactions play an important role streamlining the supply chain processes. Both individual and group human performances thus draw much attentions in the success of supply chain applications. However, little is known about the individual's contribution to the performance in the field. This study aims to understand the individuals' performance based on their individual personality traits. Attribute selection methods are used to identify the key personality traits in the Big Five Model.
\end{abstract}

\section{Keywords}

Personality Traits, Attribute Selection, Filter, Wrapper, Machine Learning

\section{INTRODUCTION}

Personality has been studied in many fields including psychology, cognitive science, behavioral science, etc. It is generally believed that personality traits provide an overall image of personality. Many studies also found personality traits to be related to human performance. Also, individuals' performances play an important role in the various supply chain management and applications. However, little is known about the factors influence human performance, especially personality traits.

This study focuses on the evaluation of individual personality traits contributing to its own performance. Following the Big Five Model, attribute selection methods are used to identify the key personality traits as factors influencing students' individual performance in a core course of supply chain management program.

\subsection{Personality Traits}

Individual's current and future performance is an important component in human resource management through the process of selection, promotion, training, and evaluation. In terms of selection, capability and personality traits were identified the indicators for future job performance (Osborne, 1998). Cattell (1943) suggested that personality can be understood by observing. As a result, interviews or personality tests have been common approaches used by employers to understand the applicant's personality, although the validity for the use of interviews to predict job performance is between 0.3 and 0.6. In addition, sometimes subjective bias issues such as interviewer's expectancy confirmation behavior may raise in interviews and therefore affect the fairness of the selection (Dougherty et al., 1994). To ensure the fairness of the selection, employers may prefer to use a systematic approach - personality test - to understand applicant's personality traits. Popular personality scales include California Psychological Inventory (CPI), MyersBriggs Type Indicator (MBTI), NEO Personality Inventory (Costa \& McCrae, 1985), Personality Characteristics Inventory (PCE), Revised NEO Personality Inventory (NEOPI-R: Costa Jr \& McCrae 2008) and so on. While the systematic approaches seem to be fair, Neal Schmitt et. al (1984) found that the average validity of personality tests was only 0.206 based on a meta-analysis of eight studies between 1962 and 1984. Therefore, the use of these personality tests for selection still remains controversial.

Although personality tests may not be the most suitable tool for selection, it was found that appropriate collection of personality data would assist the decision making on selection (Hogan 1991; Hogan \& Roberts 2001). Due to the popularity of Big Five Model, these personality tests have been adopted and tested by more researchers. Due to the advances of computing power and analytical tools, personality analysis can be quickly conducted by computers and results can be graphically displayed. The validity and reliability of these personality tests have been further enhanced.

Because of its simplicity, Saucier's Mini-Markers (1994) has been commonly utilized to measure individual's personality traits using 40 personality traits. The traits can be grouped into five areas: openness, conscientiousness, extraversion, agreeableness, and neuroticism. Table 1 shows the personality traits from the five categories of Mini-Markers proposed by Saucier. This is also the 40 traits used in the experiments in this study.

Table 1. Personality Trait Items in Mini-Markers

\begin{tabular}{|c|c|}
\hline Category & Personality Trait \\
\hline & Creative \\
& Imaginative \\
Openness & Philosophical \\
& Intellectual \\
& Complex \\
& Deep \\
& Unintellectual \\
& Uncreative \\
\hline Conscientiousness & Organized \\
& Systematic \\
\hline
\end{tabular}




\begin{tabular}{|c|c|}
\hline & $\begin{array}{c}\text { Efficient } \\
\text { Practical } \\
\text { Disorganized } \\
\text { Sloppy } \\
\text { Inefficient } \\
\text { Careless }\end{array}$ \\
\hline Extraversion & $\begin{array}{c}\text { Talkative } \\
\text { Extraverted } \\
\text { Bold } \\
\text { Energetic } \\
\text { Shy } \\
\text { Quiet } \\
\text { Bashful } \\
\text { Withdrawn }\end{array}$ \\
\hline Agreeableness & $\begin{array}{c}\text { Sympathetic } \\
\text { Warm } \\
\text { Kind } \\
\text { Cooperative } \\
\text { Cold } \\
\text { Unsympathetic } \\
\text { Harsh } \\
\text { Rude }\end{array}$ \\
\hline Neuroticism & $\begin{array}{c}\text { Touchy } \\
\text { Jealous } \\
\text { Temperamental } \\
\text { Envious } \\
\text { Moody } \\
\text { Fretful } \\
\text { Unenvious } \\
\text { Relaxed }\end{array}$ \\
\hline
\end{tabular}

\subsection{Attribute Selection}

Attribute selection is a method normally used to find top relevant factors. Not only can it identify top factors, but it also reduces dimensionality of attributes. Therefore, attribute selection is one of the most essential processes in data mining and text mining (Sebastiani 2002). There are two main types of attribute selection approaches used in the field: filter and wrapper selection approaches. The major difference between the two approaches is that the filter approach is independent of any machine learning algorithm, but the wrapper approach primarily depends on a specific machine learning algorithm to select the best subset of attributes.

In general, the filter approach evaluates attributes based on some relevance measure which is independent of any machine learning algorithm. Avrim and Pat (1997) discussed some weighting schemes that reflect how relevant each feature might be to the concept being learned. A relevance measure is normally designed as a formula to measure the dependency between the concept being learned and an attribute. The process is to keep the most relevant attributes to predicting the concept being learned, and filter out the remaining. The key benefit of the filter approach, compared to other attribute selection approaches, is its computational efficiency because the attributes need to be evaluated only once. One drawback, however, could be the selected attributes are not specifically tuned to the certain learning algorithm that is eventually employed for building the classifier (concept being learned). Another disadvantage of this filter approach is that the attributes are individually evaluated, the selected top relevant attributes may not form the best possible subset when taken together. In this study, a composite of top ranked personality traits as attributes are selected using this approach to predict individuals' performance.

On the contrary to filter approach, the wrapper approach selects a subset of the best attributes with the use of a certain learning method in predicting a class. The performance evaluation method and the search method are two major components in the wrapper approach. Cross-validation has been shown to be an effective performance evaluation method (Kohavi and John 1996; Witten and Frank 2016). This method first splits the full dataset into $k$ roughly equal-sized subsets (called folds). Next it trains a classifier based on $k-1$ subsets and evaluates it based on the remaining subset. It repeats the classifier training and evaluation $k$ times using different subsets, and then takes the average of $k$ performance results as an estimate.

Besides validation method, search method is another important component in wrapper method. Forward search and backward search are two methods that search the attribute space greedily in one of the two different directions. Forward search starts without any attribute and adds attributes one at a time until a termination condition is met. A termination condition is considered met when no new attribute leads to further performance improvement when added. Backward search method starts with a full set of attributes and keeps removing attributes one at a time until some condition is met. A termination condition is considered met when no new attribute leads to further performance improvement when removed. These two search strategies can be combined into a more sophisticated method. For instance, the best-first search method keeps an ordered list of attribute subsets evaluated until that point, and can backtrack to a previous subset when the current subset cannot be further improved.

Similar results were reached from recent studies applying various attribute selection methods. The wrapper approach has been shown to be resource demanding to large datasets with a large number of attributes (Hall and Holmes 2003). When the filter approach is adopted, information gain, gain ratio, and Chi-square have been shown to provide relatively good performance. For example, Yang and Pedersen (1997) found that information gain and Chi-square were more effective than a few other measures. Debole and Sebastiani (2004) discovered that gain ratio and Chi-square outperformed information gain. Forman (2003) reported that information gain outperformed 10 other attribute selection methods in most experiments. When time allows, the wrapper approach might provide better performance.

In order to answer the research question how individuals influence supply chain management performance, both filter and wrapper attribute selection methods were applied to identify the top relevant personability traits influencing performance. The filter attribute selection methods provide 
top ranked traits related to the results of supply chain management performance. Moreover, wrapper attribute selection methods along with several machine learning algorithms were used to identify key composite personality traits.

\section{METHODOLOGY}

Individual performance plays an important role in supply chain management due to the fact that many areas and components in supply chain require individual decisions. Better performance not only makes the process transition efficiently and effectively, but also makes the quality control more manageable.

\subsection{Data Collection}

Supply chain management performance was evaluated using a classroom setting with various of academic components covered, including operations management, quality management, statistical process control, forecasting, decision theory, reliability, enterprise resource planning. The course, offered by an ACCSB accredited school, was one of the required core courses for supply chain management education. Participants in this study were students taking this same course from multiple sections managed by the same professor. Students' performance on various academic components such as assignments, written paper, oral presentation, quizzes, and exams were evaluated by the same professor using the same standard. Moreover, students' self personality traits-- Big Five Personality Traits--were also collected in order to answer the research questions in this study. Individuals' performance were binarized into either "above average" or "below average".

The Big Five Personality Traits data were collected from each participant in early semester. The five main categories are openness, conscientiousness, extraversion, agreeableness, and neuroticism. Mini-Markers proposed by Saucier were used to obtain the personality traits. Questionnaire was distributed to 147 students and all of them participated in the study. As a results, 40 personality traits from all participants were collected.

\subsection{Attribute Selection Methods}

To identify the key factors influencing the most on individuals' performance, two attribute selection methods (Abu-Salih et al. 2018; Avrim and Pat 1997; Liu and Setiono 1995; Maini et al. 2017; Quinlan 1993) were applied on the data collected. On the one hand, filter attribute selection approach was used to rank the importance of personality traits. On the other hand, wrapper attribute selection methods along with different classifiers were utilized to find the contributing factors.

In this study, information gain, gain ratio, and Chi-squared methods were the filter attribute selection approaches applied. Decision tree (J4.8), neural network, and support vector machine were three machine learning methods used in the wrapper attribute selection approach.

\subsection{Experimental Environment}

Experiments were performed in a uniform environment. A personal computer with Intel Core i7 $4.0 \mathrm{GHz} \mathrm{CPU}$, and 16 GB 1677 MHz DDR3 RAM, running macOS Mojave 10.14.6, was used. Therefore, all results in different experiments are comparable.

The data mining tool used in this study was Weka (https://www.cs.waikato.ac.nz/ml/weka/). Weak is an open source machine learning software that provides many different data mining functionalities such as classification, clustering, attribution selection, association rules, etc. It also provides a variety of well-known algorithms. It is a Java based tool and it is customizable by adding custom algorithms for different experimental purposes.

\section{RESULTS}

When filter attribute selection methods were applied to rank the 40 personality traits relating to individuals' performance, top 10 traits ranked by information gain, gain ratio, and Chisquared were listed in Table 2.

Table 2. Top Personality Traits Ranked by Filter Method

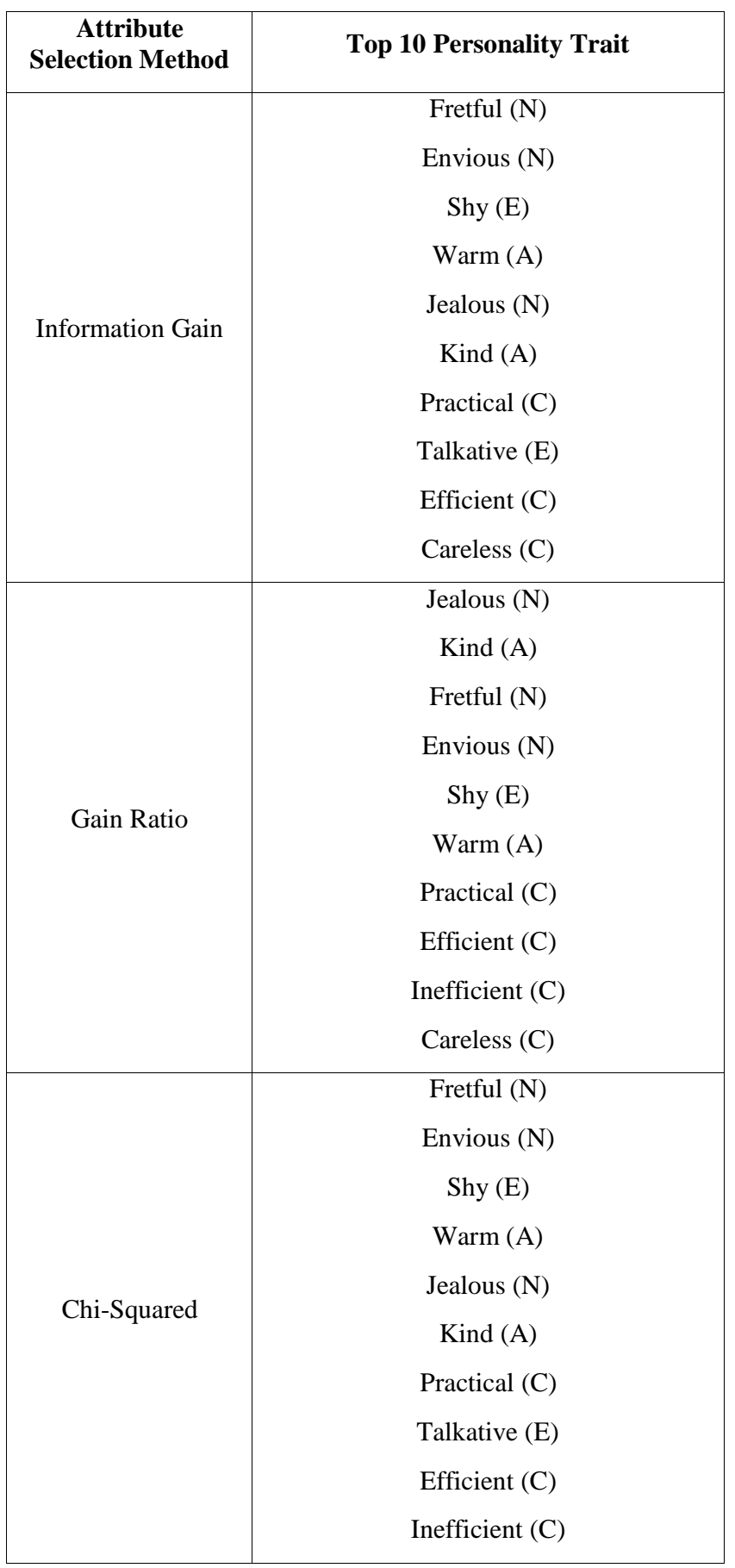


Note: the character in parenthesis refers to a Big-Five personality category: O--Openness; C--Conscientiousness; E--Extraversion; A--Agreeableness; N--Neuroticism

Information gain and chi-squared methods provided very similar top 10 personality traits, while they were quite different methods. None of openness traits were selected, inferring it not important in performance prediction. Other four categories of traits played some role influencing individuals' performance, while conscientiousness and neuroticism traits were more influential than others. The distribution of the traits is shown in Fig 1.

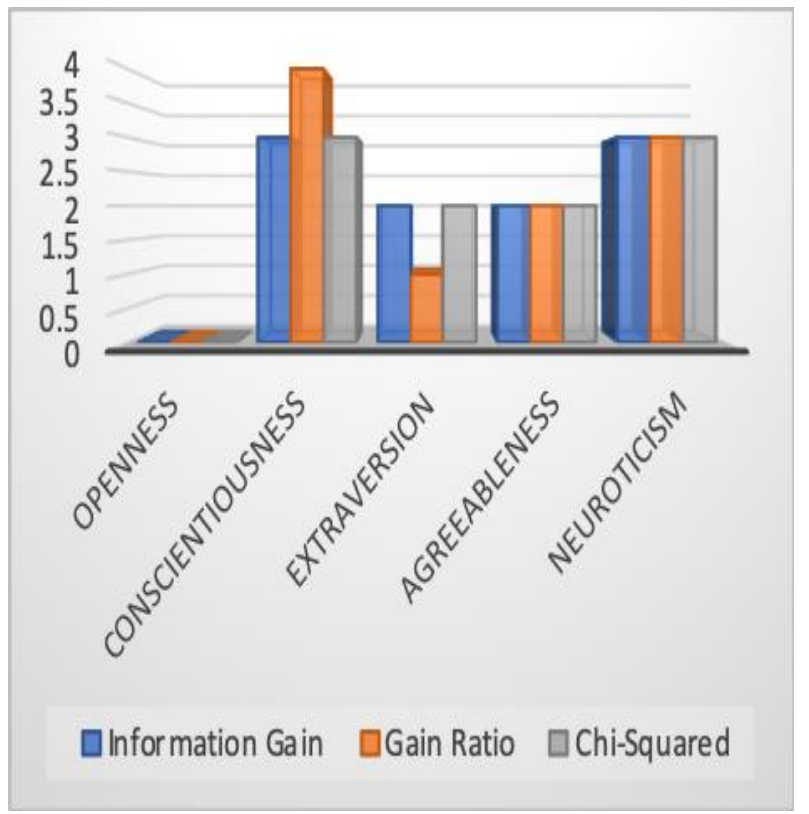

Fig 1: Distribution of Personality Traits by Filters

Unlike filter attribute selection methods which are independent on the machine learning methods, wrapper attribute selection methods are dependent on the classifiers used in evaluating individuals' performance. Decision tree (J4.8), neural network, and support vector machine were used to train classifiers. Five-fold cross-validation in wrapper was set to search the key attributes. Table 3 shows the selected attributes from the three classifiers. The distribution of the traits is shown in Fig 2.

Table 3. Personality Traits Selected by Wrapper Method

\begin{tabular}{|c|c|}
\hline $\begin{array}{c}\text { Attribute } \\
\text { Selection Method }\end{array}$ & Selected Personality Trait \\
\hline & Intellectual (O) \\
Decision Tree & Efficient (C) \\
& Envious (N) \\
\hline Neural Network & Shy (E) \\
& Quiet (E) \\
\hline Support Vector & Withdrawn (E) \\
Machine & Systematic (C) \\
\end{tabular}

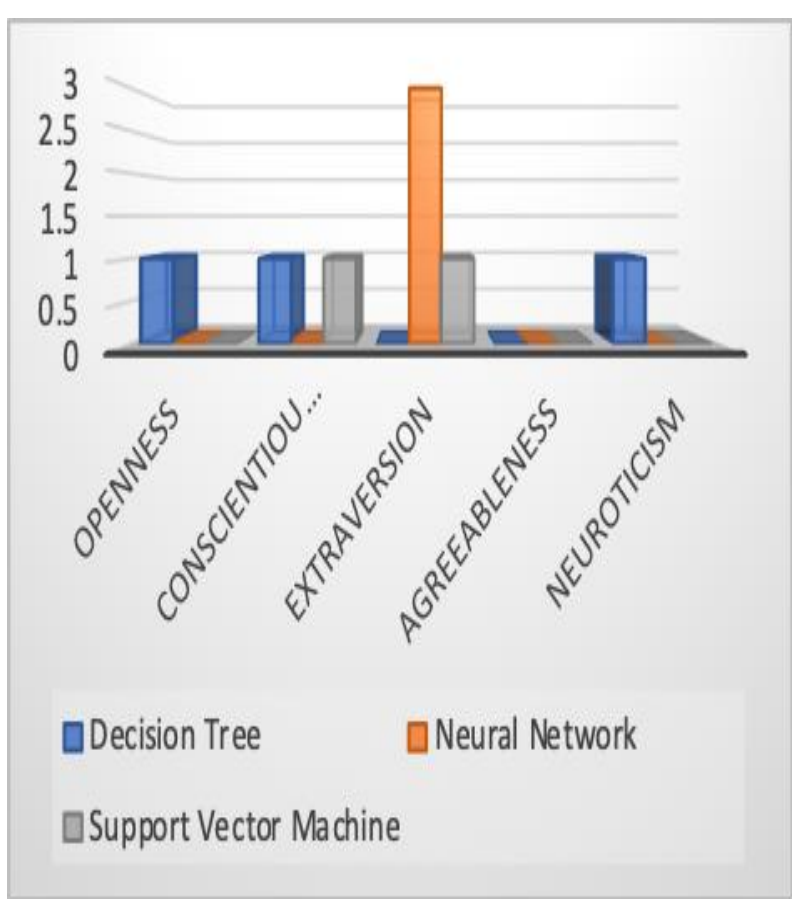

Fig 2: Distribution of Personality Traits by Wrappers

\section{CONCLUSION}

This study applied two different types of attribute selection methods to identify key personal traits influencing individuals' performance in supply chain management education. Filter attribute selection methods chose some traits in conscientiousness and neuroticism categories, while no common trait or category was identified by wrapper methods. Applying such methodology to different fields will be able to generalize a set of common traits. The contributions of this study are in two folds. To research, it demonstrates the capability of using two types of attribute selection methods in identifying key personality traits influencing individuals' performance. Practically, the traits can also be used as a reference of individuals before placements.

\section{REFERENCES}

[1] Abu-Salih, B., Wongthongtham, P. and Chan, K.Y., 2018. Twitter mining for ontology-based domain discovery incorporating machine learning. Journal of Knowledge Management.

[2] Avrim, L.B., and Pat, L. 1997. Selection of Relevant Features and Examples in Machine Learning., Artificial Intelligence (97:1-2) 1997, pp. 245-271.

[3] Cattell, R.B., 1943. The description of personality: Basic traits resolved into clusters. The journal of abnormal and social psychology, 38(4), pp. 476-506.

[4] Costa, P.T. and McCrae, R.R., 1985. The NEO personality inventory. Odessa, FL: Psychological Assessment Resources.

[5] Costa Jr, P.T. and McCrae, R.R., 2008. The Revised NEO Personality Inventory (NEO-PI-R). Sage Publications, Inc.

[6] Debole, F. and Sebastiani, F., 2004. Supervised term weighting for automated text categorization. In Text mining and its applications (pp. 81-97). Springer, Berlin, Heidelberg. 
[7] Dougherty, T.W., Turban, D.B. and Callender, J.C., 1994. Confirming first impressions in the employment interview: A field study of interviewer behavior. Journal of applied psychology, 79(5), pp. 659-663.

[8] Forman, G., 2003. An extensive empirical study of feature selection metrics for text classification. Journal of machine learning research, 3(Mar), pp.1289-1305.

[9] Hall, M.A. and Holmes, G., 2003. Benchmarking attribute selection techniques for discrete class data mining. IEEE Transactions on Knowledge and Data engineering, 15(6), pp.1437-1447.

[10] Hogan, R.T., 1991. Personality and personality measurement: handbook of industrial and organizational psychology (Editor: MD Dunnette ve LM Hough). Palo Alto.

[11] Hogan R.T. Roberts B.W. 2001. Introduction: Personality and industrial and organizational psychology. In Roberts BW, Hogan R (Eds.), Personality psychology in the workplace (pp. 3-16). Washington, DC American Psychological Association.

[12] Kohavi, R. and John, G.H., 1997. Wrappers for feature subset selection. Artificial intelligence, 97(1-2), pp. 273324.

[13] Liu, H. and Setiono, R., 1995, November. Chi2: Feature selection and discretization of numeric attributes. In Proceedings of 7th IEEE International Conference on Tools with Artificial Intelligence (pp. 388-391). IEEE.
[14] Maini, T., Kumar, A., Misra, R. and Singh, D., 2017, October. Rough set based feature selection using swarm intelligence with distributed sampled initialisation. In 2017 6th International Conference on Computer Applications In Electrical Engineering-Recent Advances (CERA) (pp. 92-97). IEEE.

[15] Osborne, R.E., 1998. Personality traits. Choice, 36, p. 223

[16] Quinlan, J.R. C4.5, 1993. Programs for machine learning Morgan Kaufmann Publishers, San Francisco, USA.

[17] Saucier, G., 1994. Mini-Markers: A brief version of Goldberg's unipolar Big-Five markers. Journal of personality assessment, 63(3), pp. 506-516.

[18] Schminke, M. and Wells, D., 1999. Group processes and performance and their effects on individuals' ethical frameworks. Journal of Business Ethics, 18(4), pp.367381

[19] Sebastiani, F., 2002. Machine learning in automated text categorization. ACM computing surveys (CSUR), 34(1), pp.1-47.

[20] Witten, I.H., and Frank, E., 2016. Data mining: practical machine learning tools and techniques, fourth edition Morgan Kaufmann Publishers.

[21] Yang, Y. and Pedersen, J.O., 1997. Proceedings of ICML-97, 14th International Conference on Machine Learning, pp. 412- 420. 